\title{
JUNÇÕES MOLECULARES DE NANOFITAS DE GRAFENO E PHAGRAPHENO: UM ESTUDO VIA DFT E EHT/NEGF *
}

\author{
Kazuko Ramos Nisioka' \\ Rafaela Farinha Felipe ${ }^{2}$ \\ Samuel Maciel Côrrea ${ }^{3}$ \\ Julio César da Silva dos Santos ${ }^{4}$ \\ Jordan Del Nero ${ }^{5}$ \\ Carlos Alberto Brito da Silva $\mathrm{Jr}^{6}$
}

\section{Resumo}

Nesse trabalho é proposto uma investigação das propriedades eletrônicas de junções moleculares de Nanofitas ZigueZague de Grafeno (NZZG) e Phagrafeno (NZZPG) por meio da Teoria: 1- DFT/B3LYP/6-311G(d,p) para otimizar a geometria das moléculas; 2- DFT/LDA.PZ/DZP sobre a célula unitária da rede cristalina para obter as propriedades eletrônicas; 3- Teoria de Hückel Estendido/Função de Green de Não Equilíbrio (THE/FGNE) através da fórmula de Landauer-Buttiker para a região central acoplada entre 2 eletrodos. Nossos resultados exibem: (i) comportamento condutor e singularidades de Van-Hove; (ii) uma região não linear com característica de um dispositivo usual TEC para NZZG; (iii) duas regiões não lineares (RDNs) mostrando característica de um diodo túnel com 2 janelas de operação para NZZPG. Essas regiões não lineares de voltagem coincidem com os $V_{\min }$ nas curvas Fowler-Nordheim (FN) e Lauritsen-Millikan (LM).

Palavras-chave: Nanofitas; Grafeno; Phagrapheno; THE/FGNE; Transistor de Efeito de Campo (TEC); Resistências Diferencial Negativa (RDNs).

\section{MOLECULAR JUNCTIONS OF GRAPHENE AND PHAGRAPHENE NANORIBBON: A STUDY BY DFT AND EHT/NEGF}

\section{Abstract}

In this work it's propose an investigation of the electronic properties of molecular junctions of ZigZag Graphene (ZZGNR) and Phagraphene (ZZPGNR) NanoRibbons by: 1- DFT/B3LYP/6-311G(d,p) for optimize the molecules; 2- DFT/LDA.PZ/DZP on the unit cell of the crystalline lattice for obtain the electronic properties; 3 - Extended Hückel Theory /Non-Equilibrium Green Function (EHT/NEGF) using the LandauerButtiker formula for the central region coupled between 2 electrodes. Our results show: (i) conductor behavior and Van-Hove singularities; (ii) a non-linear region with characteristic of a usual device FET for NZZG; (iii) two nonlinear regions (NDRs) showing behavior of a tunnel diode with 2 operation windows for ZZGNR. These nonlinear voltage regions coincide with the $V_{\min }$ in the Fowler-Nordheim (FN) and Lauritsen-Millikan (LM) plots.

Keywords: Nanoribbon; Graphene; Phagraphene; EHT/NEGF; Field Effect Transistor (FET); Negative Differential Resistances (NDRs) .

Graduanda, Faculdade de Engenharia de Materiais, UFPa, Ananindeua, Pará-Brasil. Graduanda, Faculdade de Engenharia de Materiais, UFPa, Ananindeua, Pará-Brasil. Doutorando, PPGF, Mestre, Professor, Faculdade de Ciências Exatas e Tecnologia, UFPa, Abaetetuba, Pará-Brasil.

Mestrando, PPGEE, UFPa, Belém, Pará-Brasil.

Doutor, Professor-Pesquisador, Faculdade de Física, UFPa, Belém, Pará-Brasil.

Doutor, Professor-Pesquisador, Faculdade de Física, UFPa, Ananindeua, Pará-Brasil.. 


\section{INTRODUÇÃO}

Nas duas décadas passadas, os pesquisadores tem atraído suas atenções para a investigação das propriedades eletrônica e de transporte de materiais bidimensionais (2D) de Carbono (C) feitos de apenas um átomo de espessura na camada com objetivo para aplicações eletrônicas no futuro [1]. O primeiro desses materiais, o grafeno, que foi redescoberto, isolado e caracterizado, em 2004, por A. Geim e K. Novoselov, que foram agraciados com o Prêmio Nobel de Física, em 2010, por essa conquista [2].

Devido à sua estrutura planar 2D formada por uma monocamada de átomos de $\mathrm{C}$ dispostos em uma estrutura de favo de mel hexagonal (ou colméia) onde a célula unitária da rede tem uma base de dois átomos de C, dessa forma, o grafeno apresenta propriedades únicas, tais como condutividades elétrica e térmica extremamente elevadas, velocidade alta dos elétrons e alta qualidade do cristal [35]. A maioria dos materiais pode transmitir corrente elétrica quando os elétrons não ligados $(\pi)$ têm uma energia que corresponde à banda de condução do material.

No grafeno, cada átomo de $C$ tem três elétrons que estão ligados a elétrons dos átomos vizinhos, formando ligações químicas $\sigma$ com hibridação $\mathrm{sp}^{2}$ e comprimento de ligação de 1,42 Å. O quarto elétron de cada átomo é "deslocalizado" ou livremente disponível na terceira dimensão (fora do plano) ao longo de toda a folha de grafeno, o que lhe permite conduzir a corrente elétrica. Esses elétrons altamente móveis não possuem inércia, parecem não ter massa (massa efetiva zero) e a velocidade dos elétrons no grafeno (sem dispersão, um fenômeno conhecido como transporte balístico) é de cerca de $10.000 \mathrm{~km} / \mathrm{s}$ (velocidades dos elétrons em um condutor típico variam de centímetros até centenas de metros por segundo) são chamados elétrons $\pi$ e estão localizados acima e abaixo da folha de grafeno. Esses orbitais $\pi$ se sobrepõem ajudando a realçar a ligação C-C no grafeno. Fundamentalmente, as propriedades eletrônicas de grafeno são ditadas pelos orbitais $\pi$ ligante e antiligante (as bandas de valência e condução). Entretanto, a qualidade do grafeno e do substrato usado serão os fatores limitantes. Ao mesmo tempo, a zona proíbida no grafeno tem largura zero de energia formando dois cones de Dirac conectados nos vértices dos hexágonos onde se localiza os átomos de $C$ $[2,4,5]$. Isso ocorre porque a relação energia-momentum é linear para baixas energias próximas aos seis vértices da Zona de Brillouin [5, 6].

Em 2015, Oganov et al. usando o algoritmo evolucionário ab initio USPEX (Universal Structure Predictor: Evolutionary Xtallography) descobriu um novo alotropo do grafeno feito de anéis 5-6-7 de $C$ com baixa energia denominado de phagrapheno que é um material $2 \mathrm{D}$ em que os cones de Dirac aparecem na $1^{\mathrm{a}}$ Zona de Brillouin (ZB), porém distorcidos devido ao número de átomos de $\mathrm{C}$ diferente nos anéis e os elétrons se comportam similarmente a partículas sem massa viajando com velocidade que depende da direção [7]. Esse não é o caso do grafeno. Esse alótropo de grafeno planar é energeticamente comparável ao grafeno e mais favorável do que outros alótropos do C propostos em trabalhos anteriores [8-13], devido a sua hibridização $\mathrm{sp}^{2}$ e empacotamento atômico denso. $\mathrm{O}$ phagrapheno 
possui todas as outras propriedades do grafeno que the permite ser considerado um material avançado para dispositivos eletrônicos flexíveis, transistores, baterias solares, unidades de displays, etc. [7].

Nesse sentido, baseado nos trabalhos pioneiros sobre transporte eletrônico em junções moleculares simples de Aviram-Ratner [14, 15], Reed et al. [16], Metzger et al. [17-19] e Nitzan-Ratner [20], assim bem como nos artigos publicados por nosso grupo de pesquisa [21-30] nessa área, decidimos investigar as propriedades de transporte eletrônico e Transition-Voltage Spectroscopy (TVS) de junções moleculares compostas por Nanofitas ZigueZague de Grafeno (NZZG) e Phagrapheno (NZZPG), ver Fig.1a-b, usando Teoria do Funcional Densidade (DFT) para optimizar a geometria das moléculas e Teoria de Hückel Estendido/Função de Green de Não Equilíbrio (EHT/NEGF) para o cálculo do transporte eletrônico da junção [31, 32]. A TVS é interpretada baseda em Huisman et al. [33], por meio do modelo de transporte molecular coerente pois o transporte eletrônico ocorre por níveis moleculares ocupados discretos que é descrito pela Transmitância em função da Energia em regiões onde é localizada a $V_{\min }$ [24-26]. O principal objetivo é verificar a conexão entre o transporte eletrônico e a TVS dessa classe de materiais de Dirac quase 2D por causa da higrogenização nas bordas das junções a base de NZZG e NZZPG através da Densidade de Estados (DOS), Curva I-V, Transmitância $[T(E, V)]$, curvas Fowler-Nordheim [FN ou $\left.\ln \left(\mathrm{I} \mathrm{V}^{2}\right)\right]$ e Lauritsen-Millikan [LM ou $\ln (\mathrm{I})$ ] [33-35], assim bem como a possibilidade para aplicações em nanoeletrônica.

\section{METODOLOGIA}

O formalismo DFT é altamente confiável para optimizar geometria e descrever propriedades eletrônicas de moléculas simples [36-38]. Para optimizar a geometria de NZZG e NZZPG, utilizamos cálculos de primeiros princípios baseado em DFT com functional B3LYP e conjunto de base polarizada 6-311G(d,p). Depois, escolhemos a célula unitária para a estrutura cristalina de NZZG e NZZPG que tratamos como bulk e aplicamos cálculos baseados em DFT com functional de Troca-Correlção (XC) da Aproximação de Densidade Local (LDA) com a parametrização Perdew-Zunger (PZ) [39], isto é, LDA.PZ. O conjunto de base para a Combinação Linear de Orbitais Atômicos (LCAO) escolhido foi DoubleZetaPolarized (DZP) para o Carbono e Hidrogênio assim bem como os parâmetros da iteração (density mesh cut-off $=200$ Rydberg, tolerância $=4.10^{-5}$ e passo máximo $=200$ ) enquanto que a Zona de Brillouin foi defenida com pontos $\mathrm{k}(1 \times 1 \times 100) \mathrm{e}$ condições de contorno periódico foram aplicadas para determinas as propriedades eletrônicas (estrutura de banda, DOS e Transmitância). Por outro lado, cálculos DFT são computacionalmente não viáveis para sistemas com mais de 200 átomos.

Por essa razão, tomamos a geometria optimizada (isto é, as coordenadas de todos os átomos e a célula unitária definida) que será usada para calcular o transporte eletrônico $(T(E, V))$ e TVS das junções moleculares atacadas a eletrodos (reservatórios) muito largos por meio da aproximação empírica da Teoria de Hückel Estendido (EHT) que tem sido aplicada a condução molecular [37], onde a estrutura eletrônica é calculada usando um modelo com parâmetros ajustáveis a experimentos e que combinado com a técnica da Função de Green de Não 
Equilíbrio (NEGF) para determinar o transporte quântico [38, 40,41] sob efeito de voltagem externa e que quando usados dentro das suas limitações geram resultados bem precisos. Entretanto, a principal vantagem desse método é o baixo custo computacional, mas ele captura a maioria dos efeitos eletrônicos e de estrutura atômica. Usando a aproximação EHT, sistemas com até 1000 átomos podem ser simulados facilmente [32].

A EHT é formulada em termos do conjunto de base pequenos de orbitais atômicos de Slater $\left\{\mid \phi_{i}>\right\}$, seus overlaps $\left\{S_{i j}=<\phi_{i} \mid \phi_{j}>\right\}$ e uma matriz Hamiltoniana $\left\{H_{i j}=<\phi_{i}|H| \phi_{j}\right.$ $>\}$, onde os elementos da matriz diagonal $H_{i i}=\varepsilon_{i}$ são as energias de ionização dos orbitais atômicos determinados experimentalmente e os outros elementos dessa matriz tem a forma definida por Ammeter et al. por consistência com os dados da estrutura eletrônica molecular experimental. Entretanto, os overlaps e os elementos da matriz Hamiltoniana usados nos cálculos tem a forma de onda plane no espaço-k recíproco. Logo, a equação de Schrödinger EHT pode ser resolvida [42].

Para os cálculos de transporte eletrônico, devemos especificar os eletrodos (esquerda e direita) e a região central/espalhamento, onde os eletrodos devem ser periódicos e correspondem a repetição da região de espalhamento da célula unitária da NZZG e NZZPG na direção z que é a direção do transporte eletrônico. As matrizes Hamiltoniana são construídas para os dois eletrodos dos cálculos EHT padrão sobre o dispositivo periódico bulk para a região semi-infinita. A temperatura dos elétrons é $300 \mathrm{~K}$. No início dos cálculos, a molécula relaxa livremente com a aplicação da voltagem externa e um rearranjamento estrutural da junção completa ocorre como um resultado do processo de otimização do cálculo EHT. Assim, a estrutura básica para o dispositivo é baseada sobre NZZG e NZZPG que podem ser vistas nas Figuras 1a e 1b, onde o transporte eletrônico será calculado. Nessa etapa, os cálculos são feitos com EHT e os parâmetros de entrada são definidos configurando o EHT com os parâmetros de Hoffmann e realizamos um cálculo autoconsistente para obter o espectro de transmissão, pois a transmitância é dependente de $\mathrm{k}$ para uma dada energia.

Agora, podemos calcular a corrente elétrica (curva I-V) ao aplicarmos uma voltagem externa $\left(V_{e}\right)$ finita entre os eletrodos para os dispositivos de NZZG e NZZPG. Para isso, aplicamos a técnica NEGF, pois a transmitância para cada voltagem, $T\left(E, V_{e}\right)$, é calculada após o relaxamento molecular ao aplicar $V_{e}$ variando de $0 \mathrm{~V}$ até $3 \mathrm{~V}$ para as polarizações direta e reversa entre os eletrodos. Assim, é então possível calcular a corrente (I) da região central/espalhamento de um sistema com dois eletrodos para cada voltagem aplicada por meio da fórmula de LandauerBüttiker (see Eq.1 bellow) [43, 44]

$$
I=\frac{2 e}{h} \int_{u_{E}\left(V_{e}\right)}^{u_{D}\left(V_{e}\right)} T\left(E, V_{e}\right) \cdot d E
$$

onde $u_{D}\left(V_{e}\right)$ e $u_{E}\left(V_{e}\right)$ são as regiões de polarização de energia dos eletrodos da Esquerda $(E)$ e da Direita $(R) . T\left(E, V_{e}\right)$ é o coeficiente de transmissão como função da enregia eletrônica $E$ sob voltagem externa $V_{e}$ e é dada pela Eq.(2) abaixo 


$$
T\left(E, V_{e}\right)=\operatorname{Traço}\left[\Gamma_{1}^{E} G_{C} \Gamma_{2}^{D} G_{C}^{*}\right]=\operatorname{Traço}\left[\Gamma_{1}^{E} A_{2}^{D}\right]=\operatorname{Traço}\left[\Gamma_{2}^{D} A_{1}^{E}\right]
$$

onde $G_{C}$ e $G_{C}^{+}$são as funções de Green retardada e avançada da região central (canal), $\left.\Gamma_{1 / 2}^{E / D}=i \mid \sum_{E / D}-\sum_{E / D}^{*}\right\rfloor$ é a taxa de tunelamento entre o i-ésimo nível molecular $\left(E_{i}\right) \mathrm{e}$ os eletrodos ( $D$ e $E$ ) ou funções de "alargamento" que são responsáveis pelo transporte dos elétrons no sistema e pelo alargamento dos níveis pois levam em conta o acoplamento do canal (molécula ou região central/espalhamento) com os eletrodos, $\sum_{i}^{E I D}=\tau_{E I D} g_{E / D} \tau_{E / D}^{*}$ é a função ou matriz de auto-energia dos eletrodos, $g_{E / D}=\left(E-H_{E / D}\right)^{-1}$ é a função de Green dos eletrodos e $\tau_{E / D}$ é 0 acoplamento Hamiltoniano dos eletrodos na molécula e $A_{1 / 2}^{E / D}$ são as funções espectrais parcial dos eletrodos da esquerda e da direita.

\section{RESULTADOS DA SIMULAÇÃO E DISCUSSÃO}

A configuração hexagonal do grafeno planar é obtida pelos Índices de Hamada especificado pelos inteiros $(\mathrm{M}, \mathrm{N})$ e usado para obter a estrutura do phagrapheno introduzindo defeitos topológicos como pentágonos e heptágonos na sua estrutura hexagonal, assim bem como as bordas inferior e superior do grafeno e phagrapheno são hidrogenados para formar as Nanofitas ZigueZague de Grafene (NZZG) e phagrapheno (NZZPG). A célula unitária da NZZG é composta por 12C e $2 \mathrm{H}$, enquanto a NZZPG é composta por $46 \mathrm{C}$ e $6 \mathrm{H}$. A região central/espalhamento da junção molecular da NZZG e NZZPG é composta por 10 e 3 repetições da célula unitária, respectivamente. Ao acoplar a região central/espalhamento aos eletrodos da esquerda e direita que também são compostos por 3 e 1 repetições da célula unitária, temos a junção molecular da NZZG e NZZPG efetivamente. Logo,consideramos como ponto de partida na construção separadamente da região central das junções moleculares de NZZG e NZZPG (isto é, com átomos de $\mathrm{H}$ nas bordas da configuração ziguezague) retangular com largura $=W$ (na direção armchair) e comprimento $=\mathrm{L}$ (na direção ziguezague). Dessa forma, definimos os índices de Hamada para as 2 estruturas como $M=N$, logo, $M=3$ e 2 como o número de cadeias armchair na direção armchair através das nanofitas e especificamos o parâmetro largura (W) para NZZG e NZZPG [45]. Os Índices de Hamada definem a natureza ziguezague e o comportamento metálico desses materiais de Dirac quase 2D. Quando acoplamos os eletrodos (esquerda e direita) que correspondem a repetição da região central/espalhamento da célula unitária da NZZG e NZZPG na direção z que é a direção do transporte eletrônico para formar as junções moleculares ou uma configuração de dispositivo com as dimensões determinadas na Figure 1 ( $a$ e b). 


\section{1a) Dispositivo (ou Junção Molecular) NZZG}

\section{Eletrodo Esquerdo zZGNR CenterRegion Eletrodo Direito}

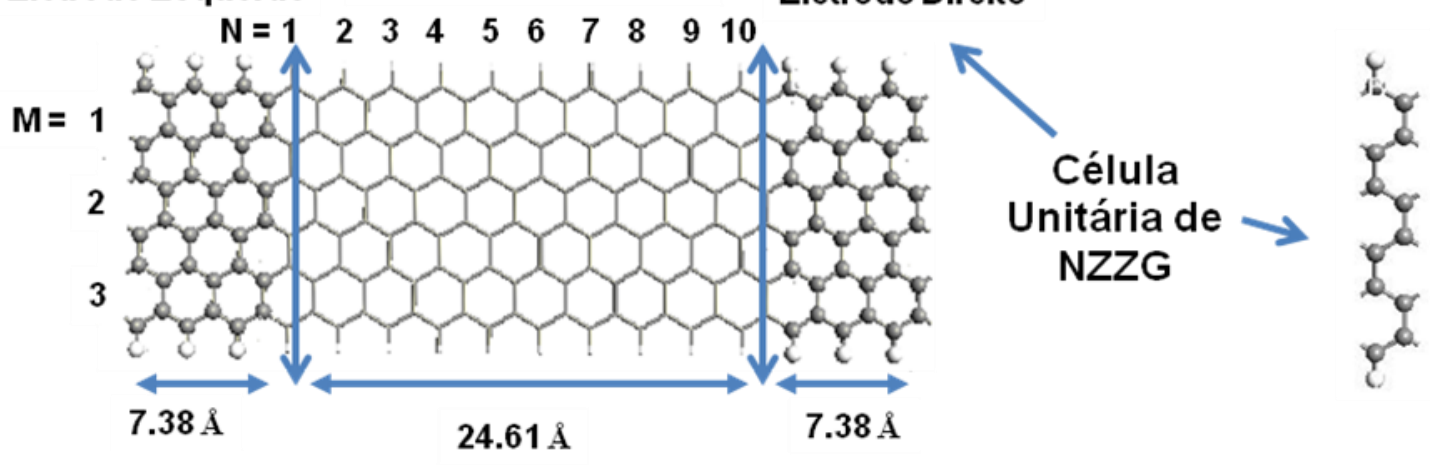

1b) Dispositivo (ou Junção Molecular) NZZPG

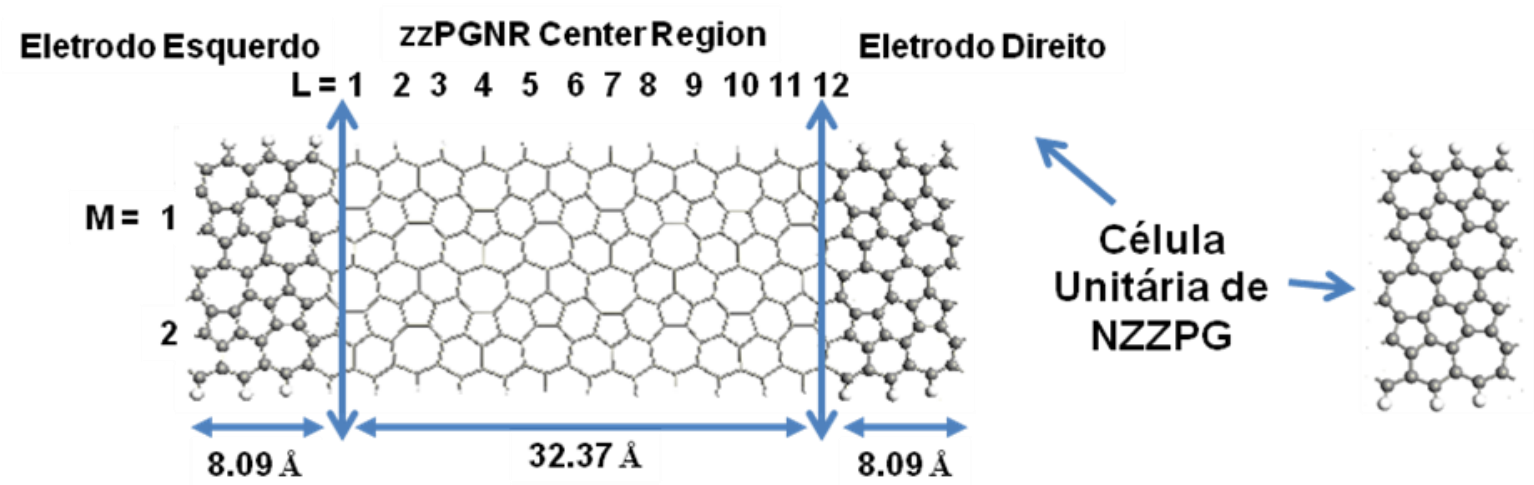

Figura 1. Estrutura de (a) NZZG e (b) NZZPG. Os índices de Hamada (M,N) para NZZG e NZZPG são $(3,3)$ e $(2,2)$. O comprimento médio das ligações são para: (a) NZZG: hexágonos $(C-C \cong 1,42 \AA$ e $\mathrm{C}-\mathrm{H} \cong 1,09 \AA \AA)$. (b) NZZPG: heptágonos $(\mathrm{C}-\mathrm{C} \cong 1,47 \AA$ e $\mathrm{C}-\mathrm{H} \cong 1,1 \AA$ ) ; hexágonos $(\mathrm{C}-\mathrm{C} \cong 1,42 \AA$ e $\mathrm{C}-\mathrm{H} \cong$ $1,09 \AA$ ); pentágonos ( $C-C \cong 1,40 \AA$ entre os anéis). A região central das NZZG e NZZPG são optimizadas por DFT/B3LYP/6-311G e tem dimensiõesn de $\cong 24,6 \AA ̊$ e $32,4 \AA ̊$. As junções moleculares são compostas pela região central acoplada aos eletrodos (E e D) onde o transporte eletrônico foi calculado por EHT/NEGF. Os eletrodos correspondem a repetição da célula unitária na região central/espalhamento para NZZG e NZZPG que foram determinadas por DFT/LDA.PZ/DPZ. Os eletrodos tem dimensões de $\cong 7,4 \AA$ e $8,1 \AA$.

$\mathrm{Na}$ Tabela 1, exibimos os resultads dos valores das energias dos Orbitais Moleculares de Fronteira $(\mathrm{HOMO}-3$ até $\mathrm{LUMO}+3)$ para as regiões centrais das NZZG e NZZPG como moléculas de tamanho finito por cálculos DFT/B3LYP/6$311 \mathrm{G}(\mathrm{d}, \mathrm{p})$, assim esses cálculos vão fazer surgir um pequeno gap na molécula que vai ser dado pelo cálculo da energia de gap ( $\mathrm{E}_{\text {gap }}=\mathrm{ELUMO}_{\mathrm{L}}$ - Еномо). O gap muito pequeno $(0,035 \mathrm{eV}$ e $0,096 \mathrm{eV})$ e, portanto, um comportamento condutor (ou metálico) para as duas moléculas (NZZG e NZZPG), respectivamente.

Tabela 1. As Energias dos OMFs via cálculo DFT/B3LYP/6-311G(d,p), a Egap = ELUMO $-E_{\text {Hомо }}$

\begin{tabular}{lcc}
\hline OMFs & $\begin{array}{c}\text { Energias p/ } \\
\text { NZZG }\end{array}$ & $\begin{array}{c}\text { Energias p/ } \\
\text { NZZPG }\end{array}$ \\
\hline HOMO-3 & $-0,31415 \mathrm{eV}$ & $-0,2856 \mathrm{eV}$ \\
\hline HOMO-2 & $-0,28992 \mathrm{eV}$ & $-0,26617 \mathrm{eV}$ \\
\hline HOMO-1 & $-0,26439 \mathrm{eV}$ & $-0,25972 \mathrm{eV}$ \\
\hline HOMO & $\mathbf{- 0 , 2 4 2 6 6 \mathrm { eV }}$ & $\mathbf{- 0 , 2 4 4 7 7} \mathrm{eV}$ \\
\hline
\end{tabular}




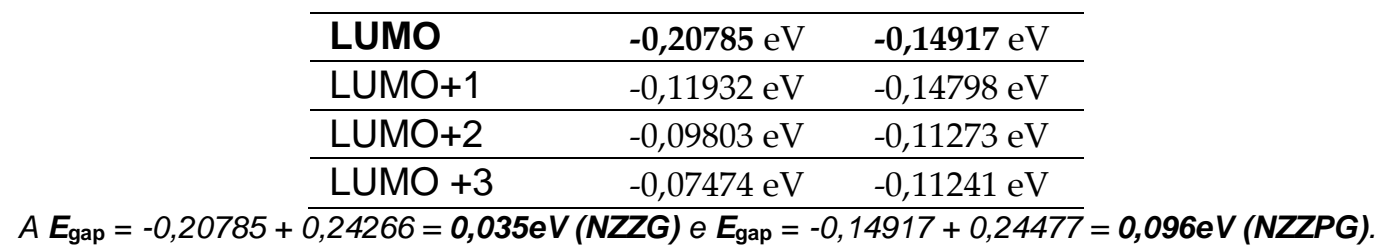

Quando o grafeno é modelado em uma nanofita reduzindo a uma dimensão (1D), existe efeitos de quantização ditados pelas condições de contorno fixadas, que podem resultar em: (1) Singularidades de Van Hove nos pontos-k de Dirac (nos vértices dos hexágonos do NZZG e nos vértices 5-6-7 dos anéis do NZZPG) para a Density de Estados (DOS) que só depende da energia e (2) um bandgap dependendo da quiralidade e da largura. Além disso, ao restringir a 1D, a dispersão da NZZG não é mais linear e, portanto perde a vantagem inerente da dispersão linear do grafeno. Na Figura 2 ( $a$ e b), exibimos as propriedades eletrônicas de NZZG e NZZP como bulk baseado na investigação da sua célula unitária no qual determinamos a estrutura de banda, DOS e transmitância em $V=0 \mathrm{~V}$ via DFT com funcional LDA.PZ e conjunto de base DZP. Esses cálculos mostraram comportamento condutor que é diferente dos resulados experimentais que mostraram comportamento semicondutor para esses materiais, pois nestes cálculos o funcional LDA não leva em consideração os spins por isso o resultado é diferente.

\section{2a) Célula Unitária NZZG $(3,3)$}
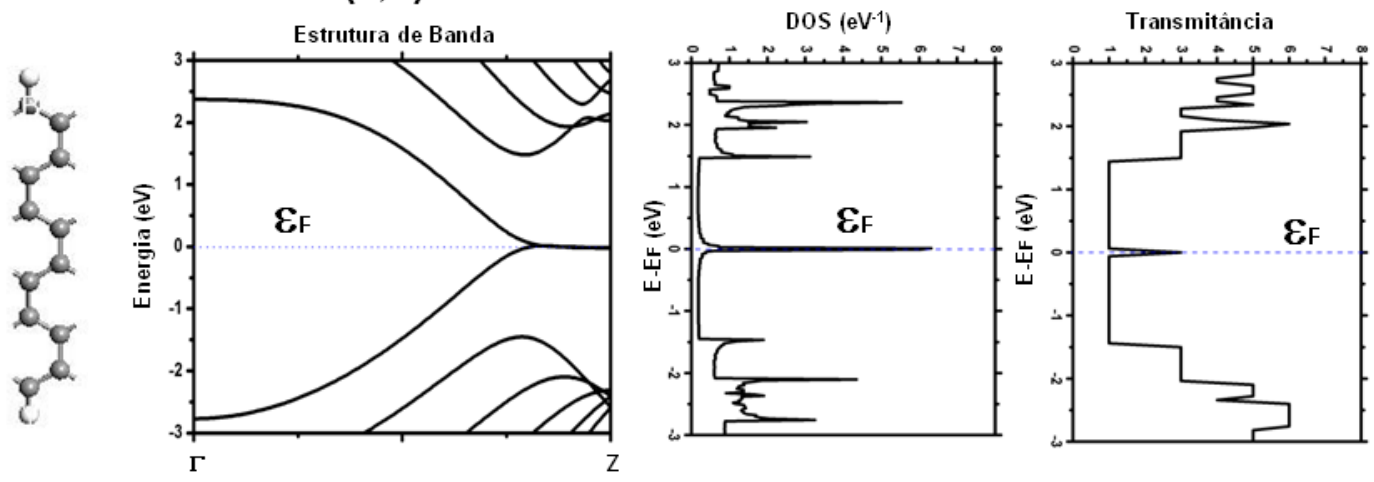

\section{2b) Célula Unitária NZZPG $(2,2)$}
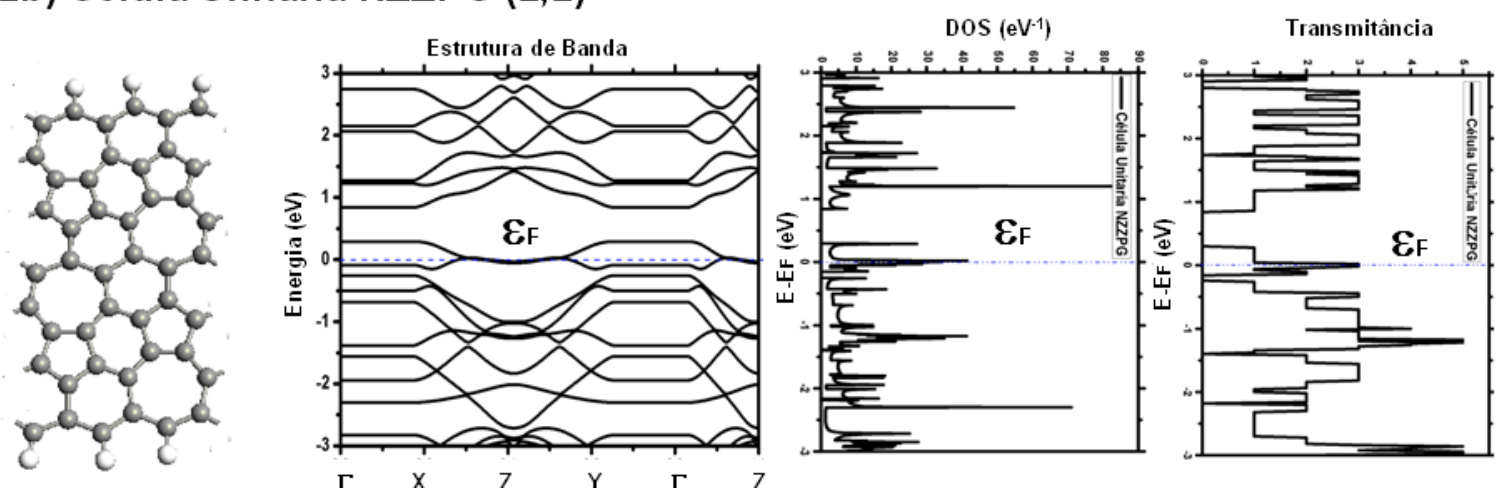

Figura 2. Propriedades Eletrônicas determinadas sobre a célula unitária de (a) $N Z Z G(3,3)$ e (b) NZZPG $(2,2)$ via DFT/LDA.PZ/DZP. A estrutura de banda e a DOS mostram que esses materiais são metálicos pois eles quase não apresentam band gap (gap muito pequeno) que é visto quando as bandas de condução (LUMOs) e valência (HOMOs) se cruzam no nível de Fermi ( $\left.E_{F}\right)$ como ocorre na NZZG ou quando ocorre uma junção das bandas na NZZPG. A DOS exibi singularidades de Van Hove que é característico de materiais quase 1D onde os átomos na NZZG e NZZPG estão localizadas. $A E_{F} \approx-3,95 \mathrm{eV}(\mathrm{NZZG})$ e $-4,36 \mathrm{eV}(\mathrm{NZZPG})$, respectivamente. A transmitância exibi 
picos de transmissão no $\mathrm{E}_{\mathrm{F}}$ e nas regiões permitidas das bandas de valência e condução. Os cálculos convergiram no passo $14 \mathrm{e} 18$, respectivamente, onde a tolerância foi de $\approx 1,03.10^{-5} \mathrm{e} 2,17.10^{-5}$.

Posteriormente, acoplamos os eletrodos (E e D) nas NZZG e NZZPG para construir efetivamente os dispositivos NZZG e NZZPG (ou junções moleculares) usando a EHT e o conjunto de base de Hückel (Cerda.Carbon [graphite] e Hoffmann. Hydrogen) para o cálculo da DOS e da Transmitância em V $=0 \mathrm{~V}$, obtendo os mesmos valores da célula unitária. Mas, a $E_{F}$ é aproximadamente $-6,26 \mathrm{eV}$ e $6,41 \mathrm{eV}$, enquanto que os potenciais químicos dos eletrodos ( $E$ e $D$ ) é de aproximadamente $-6,24 \mathrm{eV}$ e $-6,41 \mathrm{eV}$ para os dispositivos NZZG e NZZPG. A DOS em $\mathrm{V}=0 \mathrm{~V}$ mostra que eles são metálicos pois apresentam pico no nível de Fermi $\left(E-E_{F}=0\right)$ devido os níveis de energia de condução (LUMOs) e valência (HOMOs) se cruzarem nesse ponto. A Transmitância exibem picos de transmissão no nível de Fermi e nas regiões permitidas (valência e condução). Os Cálculos convergiram em 14 e 18 passos, respectivamente, assim como a tolerância era de aproximadamente $1,03.10^{-5}$ e $2,17.10^{-5}$.

Agora, exibimos na Figura 3 ( $\mathrm{a}$ e b) as propriedades de transporte eletrônico das junções moleculares por meio de calculos EHT/NEGF através das Curvas correntevoltagem (I-V) e condutância-voltagem (G-V), e das curvas Fowler-Nordheim (FN) and Lauritsen-Millikan (LM) para a escala de voltagem de $-3 \mathrm{~V} a+3 \mathrm{~V}$.
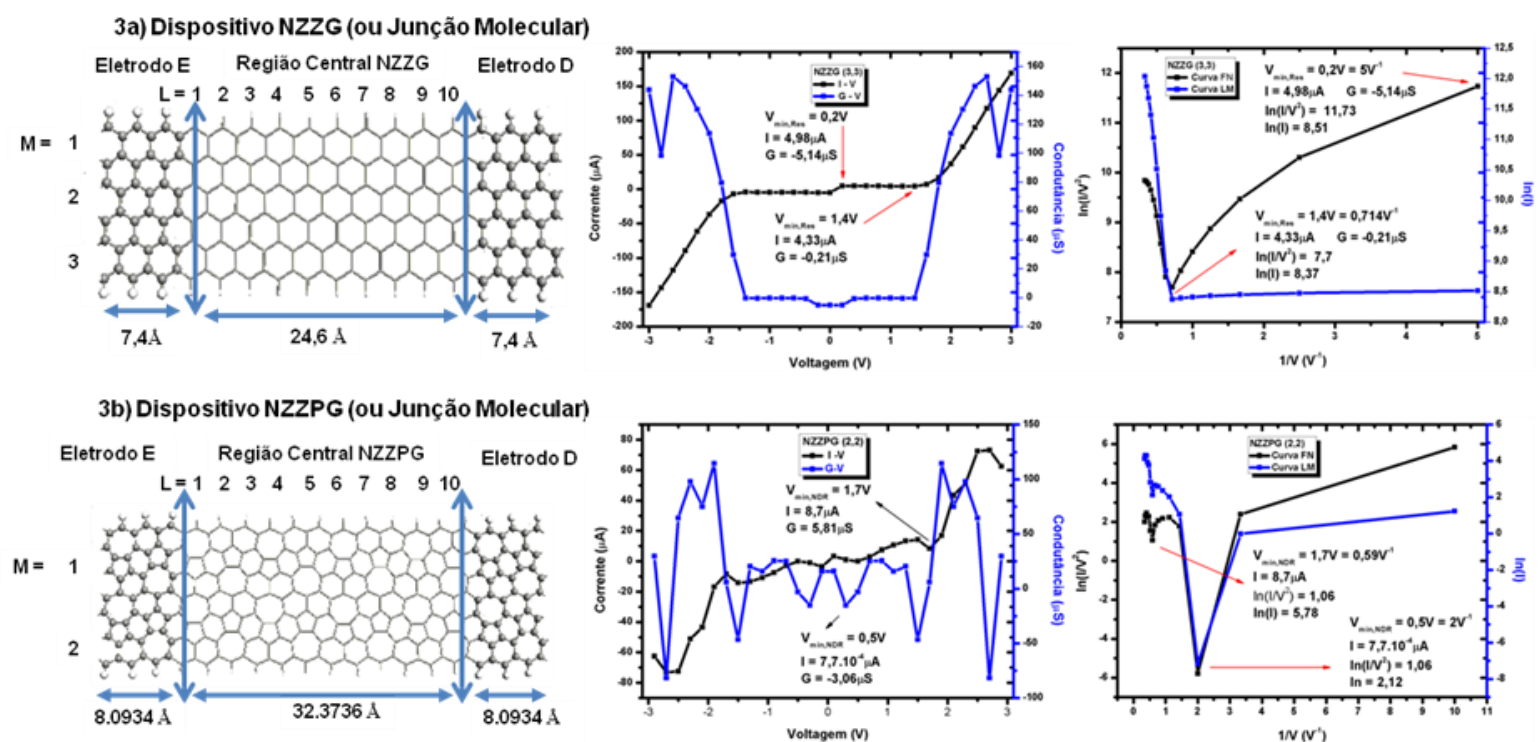

Figura 3- Transporte Eletrônico das junções determinadas por meio de cálculos EHT/NEGF. Exibimos as curvas I-V e G-V e os Plots FN e LM. As curvas I-V e G-V mostram pontos onde ocorre resonância e NDR na escala da voltagem. Esses pontos coincidem com os pontos de inflexão nos Curvas FN e LM. (a) Junção NZZG exibe duas ressonâncias enquanto em (b) Junção NZZPG exibe duas NDRs. Os cálculos convergiram em 14 e 18 passos, respectivamente, assim como a tolerância foi de aproximadamente $1,03.10^{-5}$ e $2,17.10^{-5}$.

Logo, identificamos:

1- Junção da NZZG: duas ressonâncias em $0,2 \mathrm{~V}\left(5 \mathrm{~V}^{-1}\right)$ e $1,4 \mathrm{~V}\left(0.714 \mathrm{~V}^{-1}\right)$, onde 0 valor da corrente (condutância) é $4,98 \mu \mathrm{A}(-5,14 \mu \mathrm{S})$ e $4,33 \mu \mathrm{A}(-0,21 \mu \mathrm{S})$. Em $\mathrm{V}=1,4 \mathrm{~V}$ ocorre ressonância que corresponde ao mínimo de voltagem, $\mathrm{V}_{\min }$, nos Plots de $\mathrm{FN} \mathrm{e}$ 
LM. Essa $\mathrm{V}_{\min }$ corresponde aos pontos nas curvas $\mathrm{FN}$ igual a 7,7 e LM igual a 8,05. O máximo de corrente é $I_{\max }=169,04 \mu \mathrm{A}$ em 3V. Assim, observamos que o comportamento da curva I-V é característica de um dispositivo usual (Transistor de Efeito de Campo, FET).

2- Junção da NZZPG: duas resistências diferenciais negativa (NDRs) em $0,5 \mathrm{~V}\left(2 \mathrm{~V}^{-1}\right)$ e $1,7 \mathrm{~V}\left(0,59 \mathrm{~V}^{-1}\right)$, onde 0 valor da corrente (condutância) é $7,7.10^{-4} \mu \mathrm{A}(-3,06 \mu \mathrm{S})$ e $8,37 \mu \mathrm{A}(5,81 \mu \mathrm{S})$. Esses pontos de voltagem onde ocorrem NDRs correspondem aos mínimos de voltagem, $\mathrm{V}_{\min }$, nas curvas $\mathrm{FN}$ e LM. Os $\mathrm{V}_{\min }$ correspondem aos pontos nos plots $\mathrm{FN}$ igual a $-5,78$ e 1,06 e $\mathrm{LM}$ igual a $-7,17$ e 2,12. As voltagens $0,5 \mathrm{~V}$ e $1,5 \mathrm{~V}$, correspondem aos mínimos global e local. $\mathrm{O}$ máximo de corrente é I $\max =$ $73,11 \mu \mathrm{A}$ em 2,7V. Assim, observamos que o comportamento das curvas I-V é característico de um dispositivo: (i) diodo túnel com 2 janelas de operação. A origem da NDR é devido ao alinhamento do nível de Fermi do eletrodo $\left(E_{F}\right)$ com a energia do LUMO, isto é, $E_{F}=E_{L U M O}$.

\section{CONCLUSÃO}

Nesse trabalho, investigamos as propriedades eletrônicas e de transporte eletrônico de junções moleculares baseadas em NZZG e NZZPG. Os resultados exibem para a:

(a) Junção NZZG: (1) comportamento metálico através da análise da estrutura de banda ( $E_{\text {gap }}$ próximo de $0 \mathrm{eV}(0,035 \mathrm{eV})$ cruzando as bandas de valência e condução no nível de Fermi), DOS and Transmission com picos no nível de Fermi. (2) duas ressonâncias em $0,2 \mathrm{~V}\left(5 \mathrm{~V}^{-1}\right)$ e $1,4 \mathrm{~V}\left(0.714 \mathrm{~V}^{-1}\right)$. Em $\mathrm{V}=1,4 \mathrm{~V}$, o valor da corrente é $4,33 \mu \mathrm{A}$, que corresponde ao mínimo de voltagem, $V_{\min }$, nos Plots $\mathrm{FN}$ e LM. O $\mathrm{V}_{\text {min }}$ corresponde ao ponto nas curvas $\mathrm{FN}$ igual to 7,7 e $\mathrm{LM}$ igual a 8,05. A corrente máxima é $169,04 \mu \mathrm{A}$ em $3 \mathrm{~V}$. Observamos que o comportamento da curva I-V é característico de um dispositivo usual (FET). (3) como a voltagem aumenta há um decréscimo no pico naquela região e um alargamento na $E_{\text {gap }}$ caracterizando uma transição metal-isolante que pode ser visto no LUMO que é localizado em um dos dois lados dessa junção.

(b) Junção NZZPG: (1) exibe comportamento metálico através da análise da estrutura de banda ( $E_{g a p}$, ocorre uma junção das bandas de valência e condução no nível de Fermi), DOS e Transmissão com picos no nível de Fermi. (2) duas NDRs em $\mathrm{V}_{\text {min_global }}=0,5 \mathrm{~V}\left(2 \mathrm{~V}^{-1}\right)$ com $7,7.10^{-4} \mu \mathrm{A} e$ em $\mathrm{V}_{\text {min_local }}=1,7 \mathrm{~V}\left(0,59 \mathrm{~V}^{-1}\right)$ com $8,37 \mu \mathrm{A}$ que caracteriza um diodo túnel com 2 janelas de operação. $\mathrm{O} \mathrm{V}_{\min }$ corresponde aos pontos nas curvas FN igual a $-5,78$ e 1,06 e LM igual a -7,17 e 2,12. A corrente máxima é $73,11 \mu \mathrm{A}$ em $2,7 \mathrm{~V}$. A origem da NDR é devido ao alinhamento do $\mathrm{E}_{\mathrm{F}}=$ ELUMO. (3) exibe um comportamento mais rico característico de isolante topológico que pode ser vista no HOMO e LUMO ao qual são bem delocalizado cuja superfície contém estados condutor, o que significa que os elétrons podem somente se mover ao longo da superfície da junção NZZPG, mas é um isolante no interior dessa mesma junção. 


\section{Agradecimentos}

Esse trabalho foi suportado parcialmente pelas Agências Brasileiras $\mathrm{PIBIC/UFPa/Interior,} \mathrm{CNPq,} \mathrm{CAPES,} \mathrm{FAPERJ,} \mathrm{VALE/FAPESPA,} \mathrm{Rede} \mathrm{Nanotubos}$ de Carbono/CNPq, INCT Nanomateriais de Carbono/CNPq.

\section{REFERÊNCIAS}

1 Dresselhaus M S. Fifty years in studying carbon-based Materials, Phys. Scr. 2012; T146: 014002 (1-10).

2 Novoselov KS, Geim AK, Morozov SV, Jiang D, Zhang Y, Dubonos SV et al. Electric Field Effect in Atomically Thin Carbon Films, Science 2004; 306: 666-669.

3 Dresselhaus MS e Dresselhaus G. Intercalation compounds of graphite, Adv. Phys. 2002; 51: 1-186.

4 Novoselov KS, Jiang D, Booth T, Khotkevich VV, Morozov SM e Geim AK. Twodimensional atomic crystals. Proc. Natl Acad. Sci. USA 2005; 102: 10451-10453.

5 Novoselov KS, Geim AK, Morozov SV, Jiang D, Katsnelson MI, Grigorieva IV, et al. Two-dimensional gas of massless Dirac fermions in graphene. Nature 2005; 438: 197200.

6 Wallace PR. The Band Theory of Graphite, Phys. Rev., 1947; 71: 622-634.

7 Wang Z, Zhou X.-F, Zhang X, Zhu Q, Dong H, Zhao M, et al. Phagraphene: A Lowenergy Graphene Allotrope composed of 5-6-7 Carbon Rings with Distorted Dirac Cones, NanoLetters 2015; 15: 6182-6186.

8 Malko D, Neiss C, Vines F e Görling A. Competition for Graphene: Graphynes with Direction-Dependent Dirac Cones, Phys. Rev. Lett. 2012; 108: 086804 (1-4).

9 Zhao M, Dong W e Wang A. Two-dimensional carbon topological insulators superior to graphene, Sci. Rep. 2013; 3: 3532-3537.

10 Baughman $\mathrm{RH}$, Eckhardt $\mathrm{H}$ e Kertesz M. Structure-property predictions for new planar forms of,carbon: Layered phases containing $\mathrm{sp}^{2}$ and $\mathrm{sp}$ atoms, J. Chem. Phys. 1987; 87: 6687-6699.

11 Enyashin AN e Ivanovskii AL. Graphene Allotropes, Phys. Status Solidi B 2011; 248: 1879-1883.

12 Narita N, Nagai S, Suzuki S e Nakao K. Optimized geometries and electronic structures of graphyne and its family, Phys. Rev. B. 1998; 58: 11009-11014.

13 Huang, H.; Duan, W. e Liu, Z., The existence/absence of Dirac cones in graphynes, New J. Phys. 2013; 15: 023004 (1-13).

14 Aviram A e Ratner MA. Molecular Rectifiers, Chem. Phys. Lett. 1974; 29: 277-283.

15 Aviram A e Ratner MA. Molecular Electronics: Science and Technology, Annals of the New York Academy of Sciences 1998; 852, New York.

16 Reed MA, Zhou C, Muller CJ, Burgin TP e Tour JM. Conductance of a Molecular Junction, Science 1997; 278: 252-254.

17 Metzger RM, Chen Bo, Höpfner U, Lakshmikantham MV, Vuillaume D, Kawai T, et al. Unimolecular Electrical Rectification in Hexadecylquinolinium Tricyanoquinodimethanide, J. Amer. Chem.Soc. 1997; 119: 10455-10466.

18 Metzger RM. The quest for unimolecular rectification from Oxford to Waltham to Exeter to Tuscaloosa, J. Macromol. Sci. A 2001; 38: 1499-1517.

19 Metzger RM. Unimolecular electrical rectifiers, Chem. Rev. 2003; 103: 3803-3834.

20 Nitzan A e Ratner A. Electron transport in molecular wire junctions, Science, 2003; 300: 1384-1389.

21 Nero JD, de Souza, FM e Capaz RB. Molecular Electronics Devices: Short Review, J. Comput.Theor. Nanosci. 2010; 7: 503-516.

22 Pinheiro FA, da Silva SJS, Granhen ER e Nero JD. Probing molecular chirality via electronic transport, Phys. Rev. B 2010; 81 : 115456 (1-5). 
23 Silva Jr. CAB, da Silva SJS, Granhen ER, Leal JFP, Pinheiro FA e Nero JD. Electronic transport in biphenyl single-molecule junctions with carbon nanotubes electrodes: The role of molecular conformation and chirality, Phys. Rev. B 2010; 82: 085402 (1-5).

24 Silva Jr., CAB, da Silva SJS, Leal JFP, Pinheiro FA e Nero JD. Electronic transport in oligo- para-phenylene junctions attached to carbon nanotube electrodes: Transitionvoltage spectroscopy and chirality, Phys. Rev. B 2011; 83: 245444 (1-6).

25 Silva Jr., CAB, Leal JFP, Aleixo VFP, Pinheiro FA e Nero JD. Electronic transport, transition-voltage spectroscopy, and the Fano effect in single molecule junctions composed of a biphenyl molecule attached to metallic and semiconducting carbon nanotube electrodes, Phys. Chem. Chem. Phys. 2014; 16: 19602-19607.

26 Silva Jr. CAB, Pinheiro FA e Nero JD. Organic Nano-Devices Composed by Carbon NanoTube/Oligophenylenes/Carbon NanoTube Junctions: Transition-Voltage Spectroscopy, Applications and Chirality versus Geometry, J. Nanosci. and Nanotech., 2016; 16: 9771-9778.

27 Tavares SCC, Conde-de-Sousa GT, de Sousa MÊS, Aleixo VFP e Nero JD. Electrical Signature of Graphene and Dendrimer Nanoantennas, J. Comput.Theor. Nanosci. 2014; 11: 1899-1902.

28 Sampaio-Silva, A, Aleixo VFP, Correa SM e Nero JD. Investigation of electronic transport in Organic Electroluminescent Device composed by AIQ3 molecules, J. Comput. Theor. Nanosci. 2014; 11: 1164-1168.

29 Aleixo VFP, Silva Jr. CAB e Nero JD. Molecular electronic junction composed by $\mathrm{C}_{60}$ as spacer and four terminals formed by acceptors group: Transition-voltage spectroscopy, J. Computat. and Theor. Nanosc. 2014; 11: 637-641.

30 Siqueira MRS, Corrêa SM, Gester RM, Nero JD e Neto AMJC. Single-molecular diodes based on opiod derivatives. J. Mol. Model. 2015; 21: 317 (1-9).

31 Stokbro K, Petersen DE, Smidstrup S, Blom A, Ipsen M e Kaasbjerg K. Semiempirical model for nanoscale device simulations, Phys. Rev. B 2010; 82: 075420 (1-7).

32 Kienle D, Cerda JI e Ghosh AW. Extended Hückel theory for band structure, chemistry, and transport. I.Carbon Nanotubes, J. App. Phys. 2006; 100: 043714 (1-9).

33 Huisman EH, Guédon CM, van Wees BJ e van der Molen SJ. Interpretation of transition voltage spectroscopy, Nano Lett. 2009; 9: 3909-3013.

34 Araidai $\mathrm{M}$ e Tsukada $\mathrm{M}$. Theoretical calculations of electron transport in molecular junctions: Inflection behavior in Fowler-Nordheim plot and its origin, Phys. Rev. B 2010; 81: 235114 (1-7).

35 Forbes RG. Use of Millikan-Lauritsen plots, rather than Fowler-Nordheim plots, to analyze field emission current-voltage data, J. Appl. Phys. 2009; 150: 114313 (1-8).

36 Xue, Y, Datta S e Ratner MA. First-principles based matrix Green's function approach to molecular electronic devices: general formalism, Chemical Physics 2002; 281: 151170.

37 Tian W, Datta S, Hong S, Reifenberger R, Henderson J e Kubiak CP. Conductance spectra of molecular wires J. Chem. Phys. 1998; 109: 2874-2882.

38 Di Ventra M. Electrical Transport in Nanoscale System; Cambridge University Press, 1 476; 2008.

39 Perdew JP e Zunger A. Self-interaction correction to density-functional approximations for many-electron systems, Phys. Rev. B 1981; 23: 5048-5079.

40 Cuevas JC e Scheer E. Molecular Electronics: An Introduction to Theory and Experiment, World Scientific in Nanoscience and Nanotechnology, World Scientific Publishing Co. Pte. Ltd, 1-703; 2010.

41 Zimbovskaya NA. Transport Properties of Molecular Junctions, Springer New York, 1338; 2013.

42 Raza H. Graphene Nanoelectronics: Metrology, Synthesis, Properties and Aplications, Springer Press, 1-587; 2012.

43 Landauer R. Spatial Variation of Currents and Fields Due to Localized Scatterers in Metallic Conduction, IBM J. Res. Dev. 1957; 1: 223-231. 
44 Buttiker M. Four-Terminal Phase-Coherent Conductance, Phys. Rev. Lett. 1986; 57: 1761-1764.

45 Wu C-S e Chai J-D. Electronic Properties of Zigzag Graphene Nanoribbons Studied by TAO-DFT, J. Chem. Theor. and Comput. 2015; 11:2003-2011. 